\title{
A Note on the Diffusion of Directed Polymers in a Random Environment
}

\author{
Erwin Bolthausen \\ Fachbereich Mathematik, Technische Universität Berlin, D-1000 Berlin 12
}

\begin{abstract}
A simple martingale argument is presented which proves that directed polymers in random environments satisfy a central limit theorem for $d \geqq 3$ and if the disorder is small enough. This simplifies and extends an approach by J. Imbrie and T. Spencer.
\end{abstract}

\section{Introduction}

In a recent paper, Imbrie and Spencer [1] considered the following model of a random walk in a random environment. Let $\xi(t), t \in \mathbb{N}_{0}=\mathbb{N} \cup\{0\}$ be an ordinary symmetric random walk on $\mathbf{Z}^{d}$ starting in 0 and let $h(t, x), t \in \mathbb{N}, x \in \mathbf{Z}^{d}$, be i.i.d. random variables which are + or -1 with probability $1 / 2$ and also independent of $\xi$. We denote by $\langle>$ the expectation with respect to $\xi$ and by $E($.$) the expectation$ with respect to the $h$-variables. Let $0<\varepsilon<1$ be fixed and for $T \in \mathbb{N}$,

$$
\kappa(T)=\prod_{j=1}^{T}(1+\varepsilon h(j, \xi(j))) .
$$

Imbrie and Spencer proved the following result by a rather elaborate expansion technique:

Theorem 1. If $\varepsilon>0$ is small enough and $d \geqq 3$, then

$$
\lim _{T \rightarrow \infty}\left\langle|\xi(T)|^{2} \kappa(T)\right\rangle / T\langle\kappa(T)\rangle=1 \quad \text { almost surely }
$$

(here | | is the Euclidean norm).

We give here a very simple proof based on martingale limit theorems. The result in [1] is somewhat stronger and includes also a convergence rate. Such rates can also be obtained by the method presented here. An inspection of the proof reveals that the convergence rate is $O\left(T^{-\delta}\right)$ almost surely for $\delta<(d-2) / 4$. Theorem 1 is a special case of a more general result which includes the central limit theorem which seems to be new. Let $\xi_{1}(T), \ldots, \xi_{d}(T)$ be the components of the random walk. 
Theorem 2. If $\varepsilon>0$ is small enough and $d \geqq 3$, then for all $n_{1}, \ldots, n_{d} \in \mathbb{N}_{0}$,

$$
\lim _{T \rightarrow \infty}\left\langle\prod_{j=1}^{d}\left(\frac{\xi_{j}(T)}{\sqrt{T}}\right)^{n_{j}} \kappa(T)\right\rangle\left\langle\langle\kappa(T)\rangle=\prod_{j=1}^{d} \gamma\left(n_{j}\right) d^{-n_{j} / 2} \quad\right. \text { almost surely }
$$

where $\gamma(n)=0$ if $n$ is odd, $\gamma(0)=1$, and $\gamma(2 k)=1 \cdot 3 \cdot \ldots \cdot(2 k-1)$.

This implies a central limit theorem. For a given realisation of the $h$ variables, we define the probability measure $\mu_{h}^{T}$ on $\mathbb{R}^{d}$ by

$$
\mu_{h}^{T}(A)=\left\langle 1_{A}(\xi(T) / \sqrt{T}) \kappa(T)\right\rangle /\langle\kappa(T)\rangle .
$$

Theorem 2 implies the

Corollary. For almost all $h, \mu_{h}^{T}$ converges to the centered normal law with covariance matrix $1 / d$ times the identity matrix.

\section{Proof}

Let $\mathbf{F}_{t}$ be the $\sigma$-field generated by the variables $h(s, x), s \leqq t, x \in \mathbf{Z}^{d}$.

Lemma 1. $\langle\kappa(t)\rangle$ is a nonnegative $\left(\mathbf{F}_{t}\right)$-martingale satisfying $E(\langle\kappa(t)\rangle)=1$.

Proof. $E(\langle\kappa(t)\rangle)=1$ is obvious and

$$
\begin{aligned}
E\left(\langle\kappa(t)\rangle \mid \mathbf{F}_{t-1}\right) & =(2 d)^{-t} \sum_{\substack{\omega: 0 \rightarrow \\
|\omega|=t}} E\left(\prod_{j=1}^{t}(1+\varepsilon h(j, \omega(j))) \mid \mathbf{F}_{t-1}\right) \\
& =(2 d)^{-t+1} \sum_{\substack{\omega: 0 \rightarrow \\
|\omega|=t-1}} \prod_{j=1}^{t-1}(1+\varepsilon h(j, \omega(j)))=\langle\kappa(t-1)\rangle .
\end{aligned}
$$

The summation is over nearest neighbor paths, $\omega=(\omega(0), \omega(1), \ldots, \omega(s)) . \omega: 0 \rightarrow$ stands for $\omega(0)=0$, and $|\omega|$ is the length $s$.

Lemma 2. $\langle\kappa(t)\rangle$ converges a.s. to a random variable $\zeta$ satisfying

$$
E(\zeta)=1 \text { and } P(\zeta=0)=0 \text {. }
$$

Proof. $\langle\kappa(t)\rangle$ converges a.s. by the martingale limit theorem (see e.g. [2, Theorem II-2-9]), say to $\zeta$.

We consider two independent copies of the random walk $\xi^{(1)}$, $\xi^{(2)}$ with corresponding quantities

$$
\kappa^{(i)}(t)=\prod_{j=1}^{t}\left(1+\varepsilon h\left(j, \xi^{(i)}(j)\right)\right) .
$$

The $h$ variables remain independent of $\xi^{(1)}$ and $\xi^{(2)}$. Then

$$
\begin{aligned}
E\left(\langle\kappa(t)\rangle^{2}\right) & =E\left(\left\langle\kappa^{(1)}(t)\right\rangle\left\langle\kappa^{(2)}(t)\right\rangle\right)=E\left(\left\langle\kappa^{(1)}(t) \kappa^{(2)}(t)\right\rangle\right) \\
& =\left\langle E\left(\prod_{j=1}^{t}\left(1+\varepsilon h\left(j, \xi^{(1)}(j)\right)\right)\left(1+\varepsilon h\left(j, \xi^{(2)}(j)\right)\right)\right)\right\rangle \\
& =\left\langle\left(1+\varepsilon^{2}\right)^{n_{t}\left(\xi^{(1)}, \xi^{(2)}\right)}\right\rangle,
\end{aligned}
$$


where

$$
n_{t}\left(\xi^{(1)}, \xi^{(2)}\right)=\sum_{s=1}^{t} 1_{\xi^{(1)}(s)=\xi^{(2)}(s)} \leqq n_{\infty}\left(\xi^{(1)}, \xi^{(2)}\right) .
$$

The law of $n_{\infty}$ is the same as the number of visits of a single random walk to 0 (of course, not a single nearest neighbor random walk but nevertheless, one with symmetric jump distribution). A random walk in dimension $d \geqq 3$ has after every visit to 0 a positive probability of never returning to 0 . Therefore, $n_{\infty}$ has an exponential moment. So it follows that for small enough $\varepsilon>0$,

$$
\sup _{t} E\left(\langle\kappa(t)\rangle^{2}\right)<\infty \text {. }
$$

We can conclude that $\langle\kappa(t)\rangle$ converges to $\zeta$ in $L_{2}$ and $L_{1}$ (see [2, Proposition IV$2-7])$. Therefore, $E(\zeta)=1$ and from this, we see that $P(\zeta=0) \neq 1$. It is easy to see that the event $\{\zeta=0\}$ belongs to the tail field

$$
\bigcap_{t} \sigma\left(h(s, x): s \geqq t, x \in \mathbf{Z}^{d}\right)
$$

(although $\zeta$ is certainly not tail measurable!). To see this, we write for $T>t$,

$$
\begin{aligned}
\langle\kappa(T)\rangle= & (2 d)^{-t} \sum_{x} \sum_{\substack{\omega: 0 \rightarrow x \\
|\omega|=t}} \prod_{s=1}^{t}(1+\varepsilon h(s, \omega(s)))(2 d)^{-T+t} \\
& \cdot \sum_{\substack{\omega: x \rightarrow \\
|\omega|=T-t}} \prod_{s=1}^{T-t}(1+\varepsilon h(t+s, \omega(s)))
\end{aligned}
$$

where the sum over $x$ extends to those reachable from 0 in $t$ steps. This converges to 0 for $T \rightarrow \infty$ if and only if the second part converges to 0 for any $x$ reachable from 0 in $t$ steps. Therefore, $\{\zeta=0\}$ is a tail event and by Kolmogoroffs $0-1$-law and from $P(\zeta=0) \neq 1$ it follows that $P(\zeta=0)=0$, proving the lemma.

We create now a whole family of new martingales. If $\lambda=\left(\lambda_{1}, \ldots, \lambda_{d}\right) \in \mathbb{R}^{d}$, let

$$
\varrho(\lambda)=\frac{1}{d} \sum_{j=1}^{d} \cosh \left(\lambda_{j}\right) .
$$

It is well-known (and obvious) that

$$
\exp \left(\sum_{j=1}^{d} \lambda_{j} \xi_{j}(t)-t \log \varrho(\lambda)\right)
$$

is a martingale with respect to the filtration of the random walk (no $h$-variables are involved). This remains true when $\xi(t)$ is replaced by a more general $d$-dimensional random walk $\sum_{j=1}^{t} X(j)$, where $X(j)$ are i.i.d. with $\varrho(\lambda)=\langle\exp (\lambda \cdot X)\rangle\langle\infty$ for $\lambda$ in a neighborhood of 0 in $\mathbb{R}^{d}$.

If $n=\left(n_{1}, \ldots, n_{d}\right) \in \mathbb{N}_{0}^{d}$, the polynomial $W_{n}(t, x)$ is defined by

$$
\left.\frac{\partial^{|n|}}{\partial \lambda_{1}^{n_{1}} \ldots \partial \lambda_{d}^{n_{d}}} \exp \left(\sum_{j=1}^{d} \lambda_{j} x_{j}-t \log \varrho(\lambda)\right)\right|_{\lambda=0}
$$


where $|n|=n_{1}+n_{2}+\ldots+n_{d}$. We write

$$
W_{n}(t, x)=\sum A_{n}\left(i_{1}, \ldots, i_{d}, j\right) x_{1}^{i_{1}} \ldots x_{d}^{i_{d}} t^{j}
$$

The coefficients $A$ depend on the derivatives of $\log \varrho$ in 0 .

Lemma 3. For a general random walk with $\varrho(\lambda)<\infty$ for $\lambda$ in a neighborhood of 0 and $\langle X(j)\rangle=0$, we have

a) if $i_{1}+\ldots+i_{d}+2 j>|n|$, then $A_{n}\left(i_{1}, \ldots, i_{d}, j\right)=0$.

b) The coefficients with $i_{1}+\ldots+i_{d}+2 j=|n|$ depend on the second derivatives of $\log \varrho$ at 0 .

c) If $i_{1}+\ldots+i_{d}=|n|$, then $A_{n}\left(i_{1}, \ldots, i_{d}, 0\right)=\delta_{i_{1} n_{1}} \delta_{i_{2} n_{2}} \ldots \delta_{i_{d} n_{d}}$.

Proof. a) and c) are obvious and b) follows from the fact that $\partial \varrho / \partial \lambda_{j}$ at $\lambda=0$ equals 0 .

$W_{n}(t, \xi(t))$ is a martingale for the filtration of the random walk, i.e.

$$
\left\langle W_{n}(t, \xi(t)) \mid \xi(s), s \leqq t-1\right\rangle=W_{n}(t-1, \xi(t-1)) .
$$

Here $\langle\mid \xi(s), s \leqq t-1\rangle$ denotes conditional expectation given the path up to time $t-1$. Coming back to our special symmetric random walk, it follows that

is a $\left(\mathbf{F}_{t}\right)$-martingale.

$$
Y_{n}(t)=\left\langle W_{n}(t, \xi(t)) \kappa(t)\right\rangle
$$

Lemma 4. If $|n| \geqq 1$ then

$$
\lim _{t \rightarrow \infty} t^{-|n| / 2} Y_{n}(t)=0 \quad \text { almost surely. }
$$

Proof. We show that the martingale

$$
\sum_{s=1}^{t} s^{-|n| / 2}\left(Y_{n}(s)-Y_{n}(s-1)\right)
$$

remains $L_{2}$-bounded. From this, it follows that it converges almost surely and from the Kronecker-lemma Lemma 4 follows:

$$
\begin{aligned}
& E\left(\langle W(t, \xi) \kappa(t)-W(t-1, \xi) \kappa(t-1)\rangle^{2}\right)=E\left(\langle W(t, \xi) \varepsilon \kappa(t-1) h(t, \xi(t))\rangle^{2}\right) \\
& \quad=E\left(\varepsilon^{2}\left\langle W\left(t, \xi^{(1)}\right) \kappa^{(1)}(t-1) h\left(t, \xi^{(2)}(t)\right) W\left(t, \xi^{(2)}\right) \kappa^{(2)}(t-1) h\left(t, \xi^{(2)}(t)\right)\right\rangle\right),
\end{aligned}
$$

where $\xi^{(i)}, \kappa^{(i)}$ are as in the proof of Lemma 2 and we drop the index $n$ for convenience. The above expression equals

$$
\begin{gathered}
\varepsilon^{2}\left\langle W\left(t, \xi^{(1)}\right) W\left(t, \xi^{(2)}\right)\left(1+\varepsilon^{2}\right)^{n_{t-1}\left(\xi^{(1)}, \xi^{(2)}\right)} 1_{\xi^{(1)}(t)=\xi^{(2)}(t)}\right\rangle \\
\leqq \varepsilon^{2}\left\langle W(t, \xi)^{8}\right\rangle^{1 / 4}\left\langle\left(1+\varepsilon^{2}\right)^{8 n_{\infty}\left(\xi^{(1)}, \xi^{(2)}\right)}\right\rangle^{1 / 8} P\left(\xi^{(1)}(t)=\xi^{(2)}(t)\right)^{3 / 4} . \\
P\left(\xi^{(1)}(t)=\xi^{(2)}(t)\right)^{3 / 4} \text { is of order }\left(t^{-d / 2}\right)^{3 / 4} \leqq t^{-9 / 8} \text { and } \\
\left\langle\left(1+\varepsilon^{2}\right)^{\left.8 n_{\infty}\right\rangle}\right.
\end{gathered}
$$

is finite for small enough $\varepsilon>0$. 
Therefore, in order to show that $\sup _{t} E\left(\sum_{s=1}^{t} s^{-|n| / 2}\left(Y_{n}(s)-Y_{n}(s-1)\right)\right)^{2}=\sup _{t} \sum_{s=1}^{t} s^{-|n|} E\left(\left(Y_{n}(s)-Y_{n}(s-1)\right)^{2}\right)<\infty$, it suffices to prove

$$
\left\langle W(t, \xi)^{8}\right\rangle=0\left(t^{4|n|}\right) .
$$

This is obvious from Lemma 3 a).

\section{Proof of Theorem 2}

The theorem is a consequence of Lemma 2-4. By induction, it follows from Lemma $3 \mathrm{a}), 3 \mathrm{c}$ ), and 4 that

$$
\sup _{t}\left\langle\prod_{j=1}^{d}\left(\frac{\xi_{j}(t)}{\sqrt{t}}\right)^{n_{j}} \kappa(t)\right\rangle<\infty \quad \text { almost surely . }
$$

We introduce the polynomial $U_{n}(t, x)$ by deleting from $W_{n}$ all summands

$$
A\left(i_{1}, \ldots, i_{d}, j\right) x_{1}^{i_{1}} \ldots x_{d}^{i_{d}} t^{j}
$$

with $i_{1}+\ldots+i_{d}+2 j<|n|$. We conclude from (2.1) and Lemma 4 that for $|n| \geqq 1$,

$$
\lim _{t \rightarrow \infty} t^{-|n| / 2}\left\langle U_{n}(t, \xi(t)) \kappa(t)\right\rangle=0 \quad \text { almost surely },
$$

i.e.

$$
\lim _{t \rightarrow \infty}\left\langle\sum_{i_{1}, \ldots, i_{d}} A_{n}\left(i_{1}, \ldots, i_{d}, \frac{|n|-i_{1}-\ldots-i_{d}}{2}\right)\left(\frac{\xi_{1}(t)}{\sqrt{t}}\right)^{i_{1}} \ldots\left(\frac{\xi_{d}(t)}{\sqrt{t}}\right)^{i_{d}} \kappa(t)\right\rangle=0
$$

almost surely ,

where the sum extends over those $i_{1}, \ldots, i_{d}$ with $|n|-i_{1}-\ldots-i_{d} \geqq 0$ and even. Using Lemma 2, the theorem follows by induction. This can be seen by looking at

$$
0=\frac{\partial^{|n|}}{\partial \lambda_{1}^{n_{1}} \ldots \partial \lambda_{d}^{n_{d}}}\left\langle\exp \left(\sum_{j=1}^{d} \lambda_{j} X_{j}-\frac{1}{2 d} \sum_{j=1}^{d} \lambda_{j}^{2}\right)\right\rangle
$$

where $X_{1}, \ldots, X_{d}$ are i.i.d. normally distributed random variables with mean 0 and variance $1 / d$. Because of Lemma $3 b$ ) this gives

$$
\left\langle\sum A_{n}\left(i_{1}, \ldots, i_{d}, \frac{|n|-i_{1}-\ldots-i_{d}}{2}\right) X_{1}^{i_{1}} \ldots X_{d}^{i_{d}}\right\rangle=0 .
$$

Comparing this with (2.2), the theorem follows.

Acknowledgement. I would like to thank J. Imbrie for sending me a preprint of their paper prior to publication. 


\section{References}

1. Imbrie, J.Z., Spencer, T.: Diffusions of directed polymers in a random environment. J. Stat. Phys. 52, 609 (1988)

2. Neveu, J.: Discrete parameter martingales. Amsterdam: North-Holland 1975

Communicated by J. Fröhlich

Received June 20, 1988 